\title{
A Tools-Based Approach to Teaching Data Mining Methods
}

\author{
Musa J. Jafar \\ West Texas A\&M University \\ Canyon, TX, USA
}

\section{mjafar@mail.wtamu.edu}

\section{Executive Summary}

Data mining is an emerging field of study in Information Systems programs. Although the course content has been streamlined, the underlying technology is still in a state of flux. The purpose of this paper is to describe how we utilized Microsoft Excel's data mining add-ins as a front-end to Microsoft's Cloud Computing and SQL Server 2008 Business Intelligence platforms as backends to teach a senior level data mining methods class. The content presented and the hands on experience gained have broader applications in other areas, such as accounting, finance, general business, and marketing. Business students benefit from learning data mining methods and the usage of data mining tools and algorithms to analyze data for the purpose of decision support in their areas of specialization.

Our intention is to highlight these newly introduced capabilities to faculty currently teaching a business intelligence course. Faculty interested in expanding their teaching portfolio to the data mining and the business intelligence areas may also benefit from this article.

This set of integrated tools allowed us to focus on teaching the analytical aspects of data mining and the usage of algorithms through practical hands-on demonstrations, homework assignments, and projects. As a result, students gained a conceptual understanding of data mining and the application of data mining algorithms for the purpose of decision support. Without such a set of integrated tools, it would have been prohibitive for faculty to provide comprehensive coverage of the topic with practical hands-on experience.

The availability of this set of tools transformed the role of a student from a programmer of data mining algorithms to a business intelligence analyst. Students now understand the algorithms and use tools to perform (1) elementary data analysis, (2) configure and use data mining computing engines to build, test, compare and evaluate various mining models, and (3) use the mining models to analyze data and predict outcomes for the purpose of decision support. If it was not for the underlying technologies that we used, it would have been impossible to cover such material in a one-semester course and provide students with much needed hands-on experience in data mining.

Material published as part of this publication, either on-line or in print, is copyrighted by the Informing Science Institute. Permission to make digital or paper copy of part or all of these works for personal or classroom use is granted without fee provided that the copies are not made or distributed for profit or commercial advantage AND that copies 1) bear this notice in full and 2) give the full citation on the first page. It is permissible to abstract these works so long as credit is given. To copy in all other cases or to republish or to post on a server or to redistribute to lists requires specific permission and payment of a fee. Contact 0HPublisher@InformingScience.org to request redistribution permission.
Finally, what we presented is how to utilize the cloud as a computing platform that transformed the role of a student from "doing low-level IT" in a data mining course to a business intelligence analyst using tools to analyze data for the purpose of decision support. 
Keywords: Data mining, Decision Support, Business Intelligence, Excel Data mining Add-ins, Cloud Computing.

\section{Introduction}

Data mining is the process of discovering useful and previously unknown information and relationships in large data sets (Campos, Stengard, \& Milenova, 2005; Tan, Steinbach, \& Kumar, 2006). Accordingly, data mining is the purposeful use of information technology to implement algorithms from machine learning, statistics, and artificial intelligence to analyze large data sets for the purpose of decision support.

The field of data mining grew out of limitations in standard data analysis techniques (Tan et al., 2006). Advancements in machine learning, pattern recognition, and artificial intelligence algorithms coupled with computing trends (CPU power, massive storage devices, high-speed connectivity, and software academic initiatives from companies like Microsoft, Oracle, and IBM) enabled universities to bring data mining courses into their curricula (Jafar, Anderson, \& Abdullat, 2008b). Accordingly, Computer Science and Information Systems programs have been aggressively introducing data mining courses into their curricula (Goharian, Grossman, \& Raju, 2004; Jafar, Anderson, \& Abdullat 2008a; Lenox \& Cuff, 2002; Saquer, 2007).

Computer Science programs focus on the deep understanding of the mathematical aspects of data mining algorithms and their efficient implementation. They require advanced programming and data structures as prerequisites for their courses (Goharian et al., 2004; Musicant, 2006; Rahal, 2008).

Information Systems programs on the other hand, focus on the data analysis and business intelligence aspects of data mining. Students learn the theory of data mining algorithms and their applications. Then they use tools that implement the algorithms to build mining models to analyze data for the purpose of decision support. Accordingly, a first course in programming, a database management course, and a statistical data analysis course suffice as prerequisites. For Information Systems programs, a data centric, algorithm understanding and process-automation approach to data mining similar to Jafar et al. (2008a) and Campos et al. (2005) is more appropriate. A data mining course in an Information Systems program has an (1) analytical component, (2) a toolsbased, hands-on component, and (3) a rich collection of data sets.

(1) The analytical component covers the theory and practice of the lifecycle of a data mining analysis project, elementary data analysis, market basket analysis, classification and prediction (decision trees, neural networks, naïve Bayes, logistic regression, etc.), cluster analysis and category detection, testing and validation of mining models, and finally the application of mining models for decision support and prediction. Textbooks from Han and Kamber (2006) and Tan et al. (2006) provide a comprehensive coverage of the terminology, theory, and algorithms of data mining.

(2) The hands-on component requires the use of tools to build projects based on the algorithms learned in the analytical component. We chose Microsoft Excel with its data mining add-in(s) as the front-end and Microsoft's Cloud Computing and SQL Server 2008 data mining computing engines as the back-end. Microsoft Excel is ubiquitous. It is a natural front-end for elementary data analysis and presentation of data. Its data mining add-in(s) are available as a free download. The add-in(s) are automatically configured to send data to Microsoft's Cloud Computing engine server. The server performs the necessary analysis and receives analysis results back into Excel to present them in tabulated and chart formats. Using wizards, the add-in(s) are easily configured to connect to a SQL Server 2008 running analysis services to send data and receive analysis results back into Excel for presentation. The add-in(s) provide a rich wizard-based, uniform graphical user interface to manage the data, the data mining models, the configurations, and the pre and 
post view of data and mining models. They provide a workbench for an end-to-end life-cycle support of a data mining project.

(3) A rich collection of data sets to demonstrate the capabilities of the algorithms and to provide practical hands-on experience, homework assignments and projects is vital. We chose to use the Iris data set, the Mushrooms data set, and the Bikebuyers data set. The Iris and the Mushrooms data sets are public domain data sets available from the UCI repository (University of California Irvine, 2009). In this paper, we use the Iris and the Mushrooms data sets for elementary data analysis, classification, and clustering analysis. The Iris data set attributes are quantitative. It is composed of 150 records of: Iris(sepalLength, sepalWidth, petalLength, petalWidth, irisType) for a total of 4 attributes in each record. The length and width attributes are in $\mathrm{cm}$ and the classification (iris-type) is setosa, versicolor, or virginica. The Mushrooms data set attributes are qualitative. It is composed 8,124 records of: Mushroom(capShape, capSurface,..., odor, ringType, habitat, gillSize, ......, classification) for a total of 21 attributes in each record. The classification of a mushroom is either poisonous or edible. The Bikebuyers data set is available from Microsoft Corporation in support of their Business Intelligence set of tools. We will use this data set for market basket analysis. The data set is composed of 121,300 records of: Bike-

Buyer(salesOrderNumber, quantity, productName, model, subcategory, category) for a total of 6 attributes in each record.

\section{The Data Mining Process}

Given a data set, the data mining process starts with elementary data analysis. It allows an analyst to understand the characteristics of the attributes of the data set (dependency, ranges, max, min, count, average, standard deviation, quartiles, outliers, etc.). The data set is then divided into a training data set and a testing and validation data set (holdout). The training data set is used to build the mining structure and associated mining models. Using accuracy charts, the holdout set is used to test the accuracy and the efficiency of the mining models. If a model is valid and its accuracy is acceptable, it is then used for prediction (Figure 1). The data mining process is very elabo-

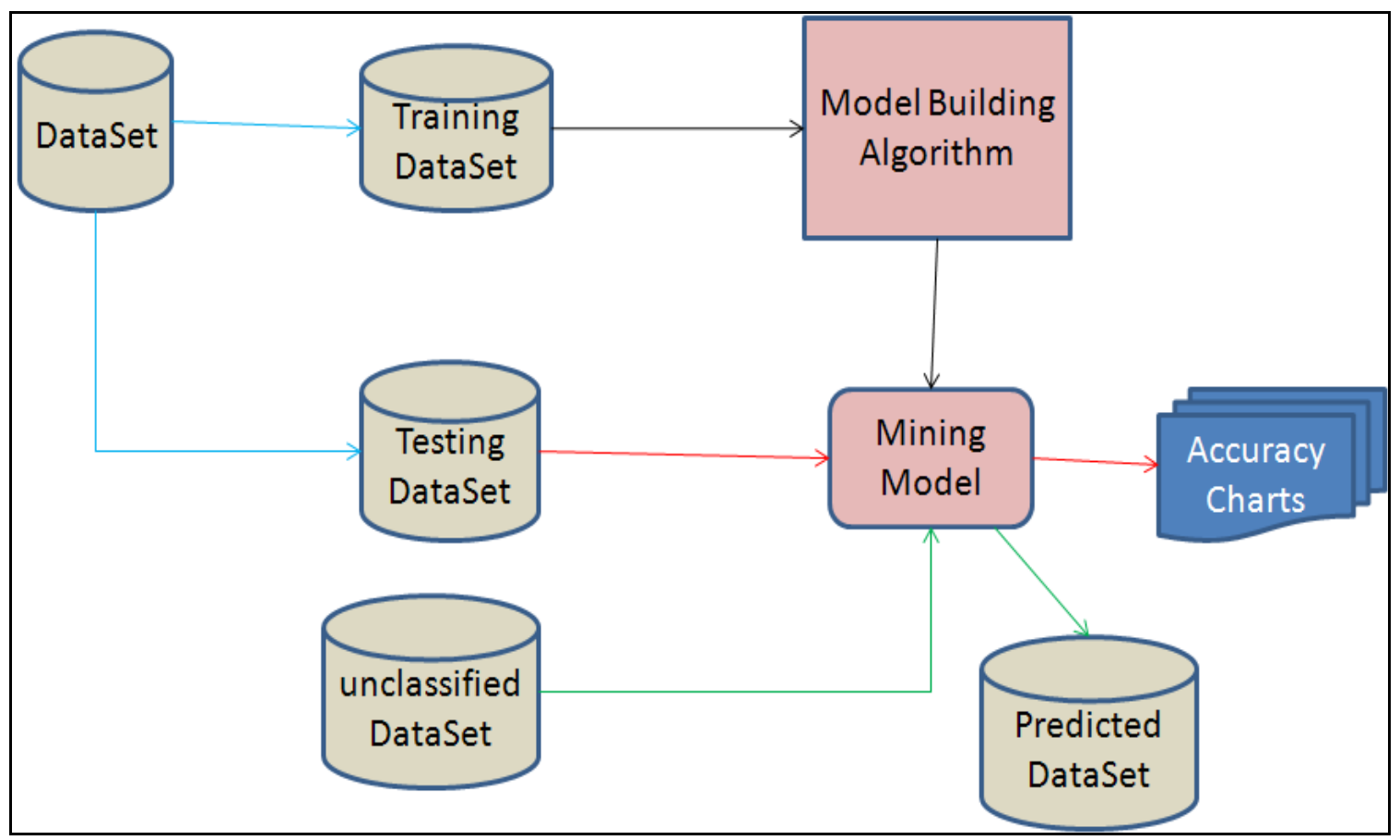

Figure 1: High Level Flow of Data Mining Activities 
rate. It requires extensive front-end processing and presentation of results. In the past, our students had to write their own macro(s) to randomly split a data set into training and testing sets. With Excel's data mining add-ins, this task is performed automatically through configuration. It allowed both faculty and students to focus on the configuration and analysis tasks instead of writing random number generator macros to split the data.

For the rest of the paper, we will take a hands-on approach to data mining with examples. Each section will be composed of a theory component of data mining followed by a practice component. We will review the basic topics taught in a standard data mining course. These topics are (1) elementary data analysis and outlier detection, (2) market basket analysis or association rules analysis, (3) classification and prediction, and (4) clustering analysis. The last section of the paper is a summary and conclusions section. The topics, subtopics and, terminology used can be found in standard data mining text books such as Han and Kamber (2006) and Tan et al. (2006).

\section{Elementary Data Analysis}

Elementary data analysis is the first basic step in data mining. It allows an analyst to understand the intricacies of a data set, the characteristics of each attribute, and the dependencies between attributes.

\section{The Theory}

Students learn the concepts of elementary data analysis through book chapters, lecture notes, lectures, and homework assignments. Students learn to: (1) Classify the data type of each attribute (quantitative, qualitative, continuous, discrete, or binary) and its scale of measure (nominal, ordinal, interval, or ratio); (2) Produce summary statistics for each quantitative attribute (mean, median, mode, min, max, quartiles, etc.); (3) Visualize data through histograms, scatter plots, and box plots; (4) Produce hierarchical data analysis through pivot tables and pivot charts; (5) Produce and analyze the various correlation matrices and key influencers of attributes; (6) In preparation for data mining, attributes and their values may also need to be relabeled, grouped, or normalized.

\section{The Practice}

The hands-on practice of elementary data analysis is performed in Excel. Most of the elementary data analysis tasks can be performed in Excel's charting, sorting, tables, and pivot tables functions (King, 2009; Tang, 2008). The data analysis add-in tools allow students to generate descriptive statistics, correlation matrices, histograms, percentiles, and scatter plots. Using wizards, a student can easily produce summary statistics similar to those in Figures 2, 3, and 4.

In Excel, the table, pivot table, and charts tools allow students to perform various hierarchical analyses and relabeling of data. Elementary data analysis of the Iris data set is shown in Figure 3. It is easy to see that all petal lengths, petal widths, and sepal lengths of the setosa(s) are at the low end of the scale. The petal lengths and petal widths of the virginica(s) are at the high end of the scale. Filters can also be added on top of a row or column contents to produce hierarchical representation of the data to provide more resolution during data analysis. 


\begin{tabular}{|c|c|c|c|c|}
\hline & Sanallanyth & Sopallitith & Fet.alLength & 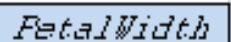 \\
\hline Mean & 5.84 & 3.05 & 3.76 & 1.20 \\
\hline Standard Error & 0.07 & 0.04 & 0.14 & 0.06 \\
\hline Median & 5.80 & 3.00 & 4.35 & 1.30 \\
\hline Mode & 5.00 & 3.00 & 1.50 & 0.20 \\
\hline Standard Deviation & 0.83 & 0.43 & 1.76 & 0.76 \\
\hline Sample Variance & 0.69 & 0.19 & 3.11 & 0.58 \\
\hline Kurtosis & -0.55 & 0.29 & -1.40 & -1.34 \\
\hline Skewness & 0.31 & 0.33 & -0.27 & -0.10 \\
\hline Range & 3.60 & 2.40 & 5.90 & 2.40 \\
\hline Minimum & 4.30 & 2.00 & 1.00 & 0.10 \\
\hline Maximum & 7.90 & 4.40 & 6.90 & 2.50 \\
\hline Sum & 876.50 & 458.10 & 563.80 & 179.80 \\
\hline Count & 150.00 & 150.00 & 150.00 & 150.00 \\
\hline Conf idence Level (95) & 0.13 & 0.07 & 0.28 & 0.12 \\
\hline & Sanallendth & 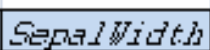 & 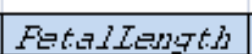 & 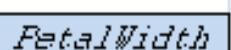 \\
\hline SepalLength & 0.68 & & & \\
\hline SepalWidth & -0.04 & 0.19 & & \\
\hline Petallength & 1.27 & -0.32 & 3.09 & \\
\hline PetalWidth & 0.51 & -0.12 & 1.29 & 0.58 \\
\hline & & & & \\
\hline
\end{tabular}

Figure 2: Descriptive Statistics and Correlation Matrix Results from Excel

Using Excel's data mining add-ins, we can also analyze the overall key influencers of an iris type. We can measure the relative impact of each attribute value on the classification of an iris (Figure 4). We can also perform pair wise comparisons between the different classifications. Based on an analytical model, the key influencers tool automatically breaks the range of a continuous attribute into intervals while determining the influencers of the iris type. The algorithm discovered that a petalWidth $<0.4125$ strongly favored the setosa classification. A petalWidth in the range of $[0.4125,1.33]$ strongly favored a versicolor classification and a petalLength $>=5.48$ strongly favored a virginica classification. A student can use this visual analysis and presentation of the key influencers to build the initial classification rules for an expert system. Excel tools allow students to perform pair wise discrimination for key influencers of the different types of classifications. The length of the "Relative Impact" bar indicates the relative importance of each attribute range to the corresponding classification (Figure 4).

Excel's data exploration tool allows users to interactively produce histograms and configure bucket counts. The data clean-up tool permits users to interactively create line charts and specify ranges for outliers of numeric data. With the data sampling tool users interactively divide the data into different random samples. Users are able to interactively re-label data into ranges such as low, medium, high with the re-labeling tool. 


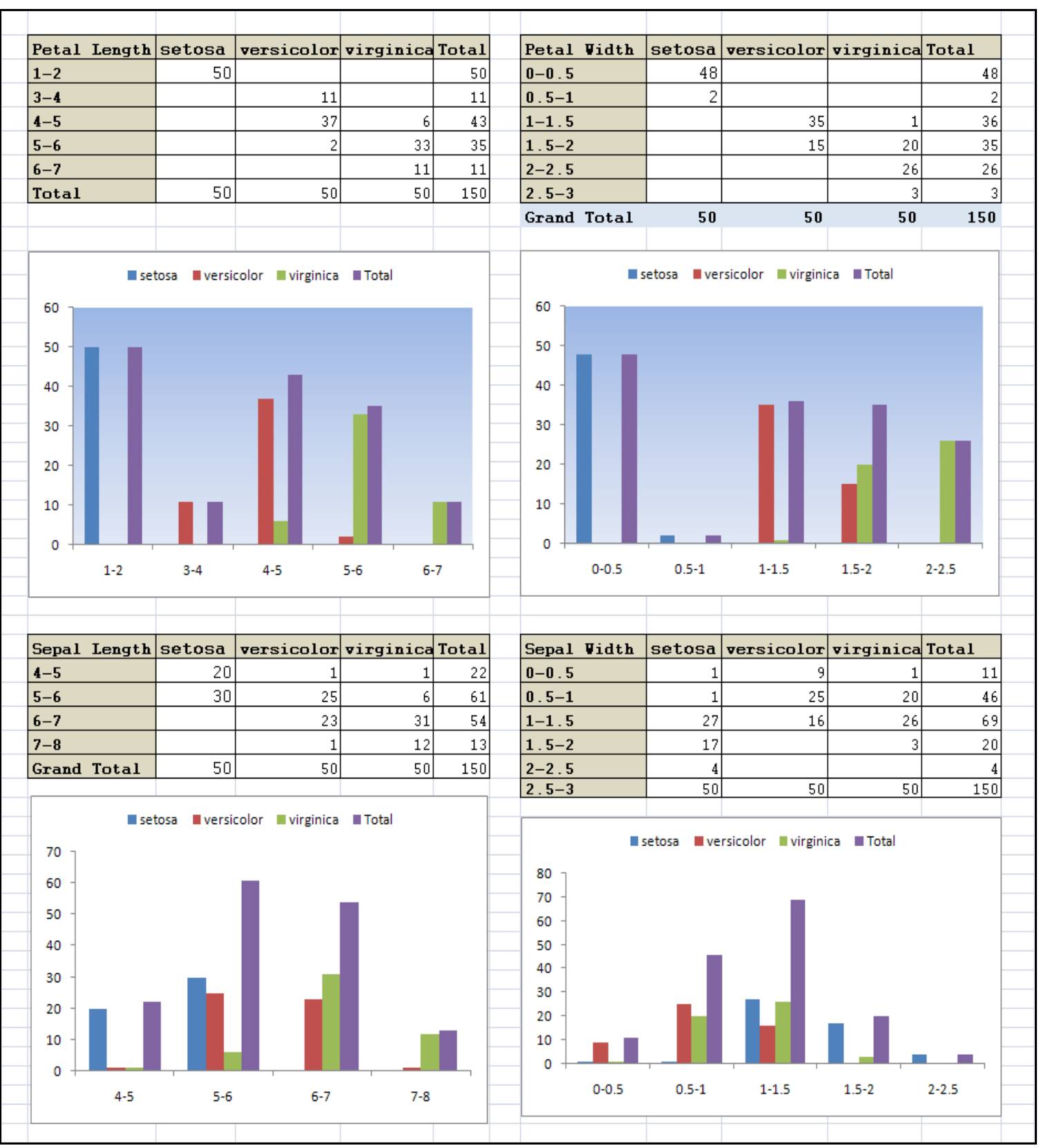

Figure 3: Pivot Tables and Analysis Charts 


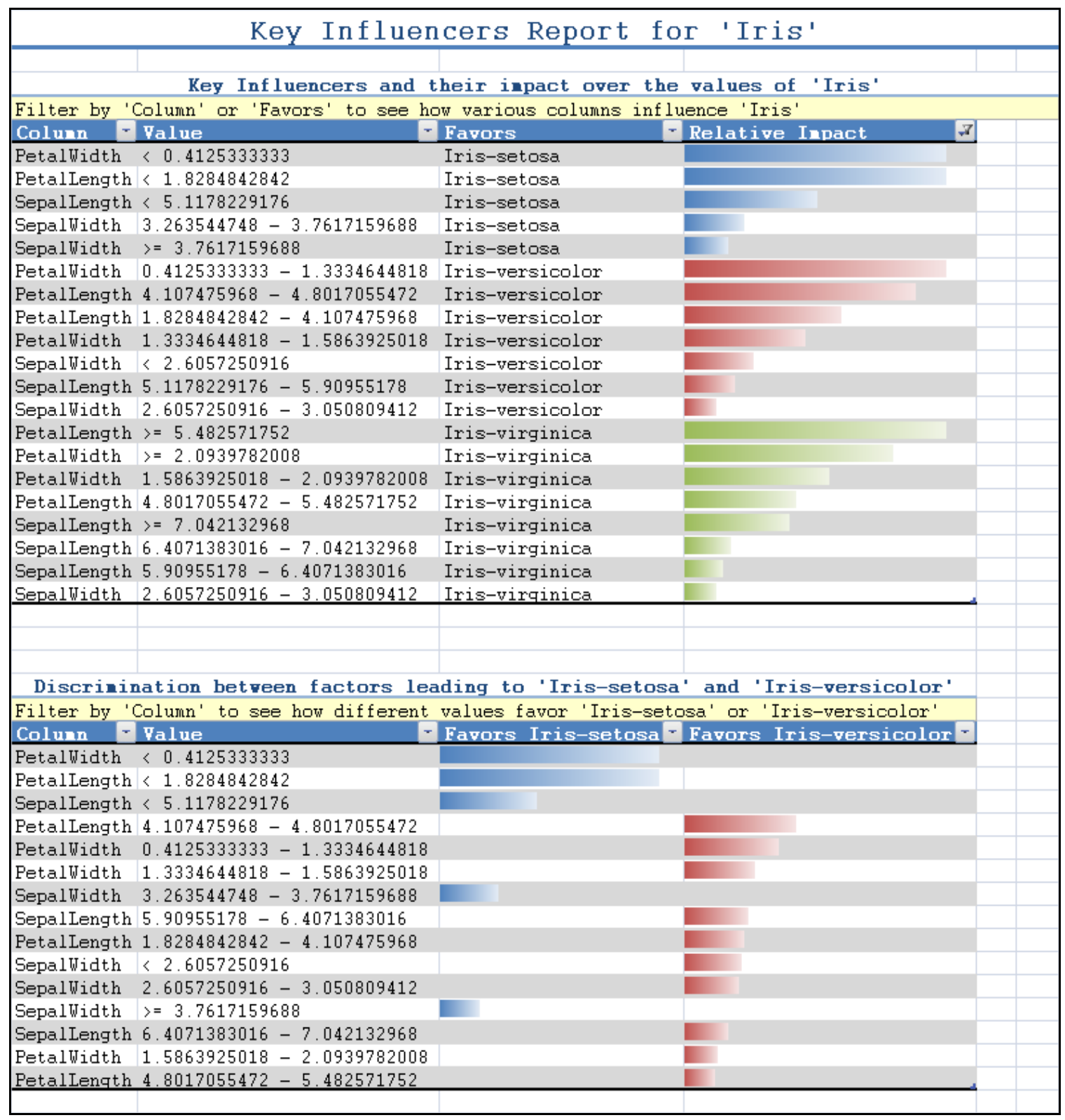

Figure 4: Key Influencers Analysis Results

\section{Market Basket Analysis}

Market basket analysis allows a retailer to understand the purchasing behavior of customers. It predicts products that customers may purchase together. It allows retailers to bundle products, offer promotions on products or suggest products that have not yet been added to the basket. Market basket analysis can be used to analyze the browsing behavior of students inside a course management system by modeling each visit as a market basket and the click stream of a student as a set of items inside a basket.

\section{The Theory}

Students learn the theoretical foundations and concepts of association analysis through book chapters, lecture notes, lectures, and homework assignments. Students learn conditional probabil- 
ity concepts and Bayesian statistics, the concepts of item sets, item set support and its calculation, frequent item sets, closed frequent item sets, association rules, rule support and its calculations, rule confidence and its calculations, rule strength and its calculations, rule importance and its calculations, correlation analysis and the lift of association rules and their calculations, apriori and general algorithms for generating frequent item sets from a market basket set, apriori and general algorithms for generating association rules from a frequent item set. Given a small market basket and a set of thresholds, students should be able to use algorithms to manually (apriori and confidence-based pruning) generate the pruned item set, detect closed item sets, generate rules and calculate their support, and the confidence and importance of rules as shown in the activity diagram in Figure 5. The cited textbooks present algorithms in complex English-like structures with mathematical notations. It is more helpful to students when faculty visually present an algorithm by flowcharting it as a UML-based activity diagram and then use an example to demonstrate the algorithm in action. Figure 5 is an activity diagram of the apriori algorithm for discovering fre-

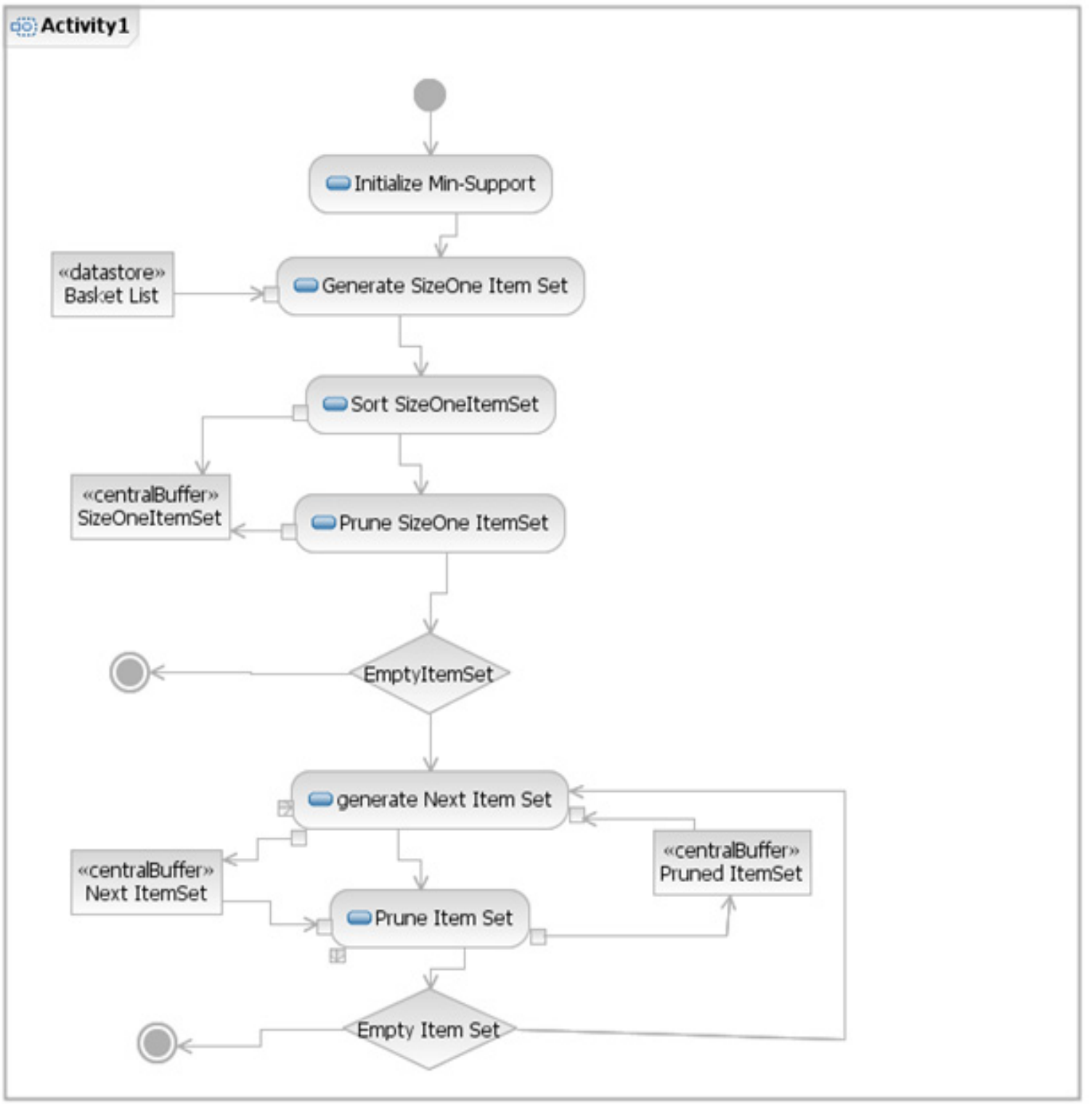

Figure 5: An Activity Diagram of the Apriori Item Set Generation Algorithm 
quent item sets (item sets with two or more items). Figure 6 is an example implementation of the algorithm as it applies to an item set of purchases. In Figure 6 we started with 6 transactions and then used the apriori algorithm to generate all the frequent item sets with a minimum support of 2 . We stopped when no item sets with the minimum threshold support could be generated. Item sets with support less than 2 (the red item sets) were successively eliminated.

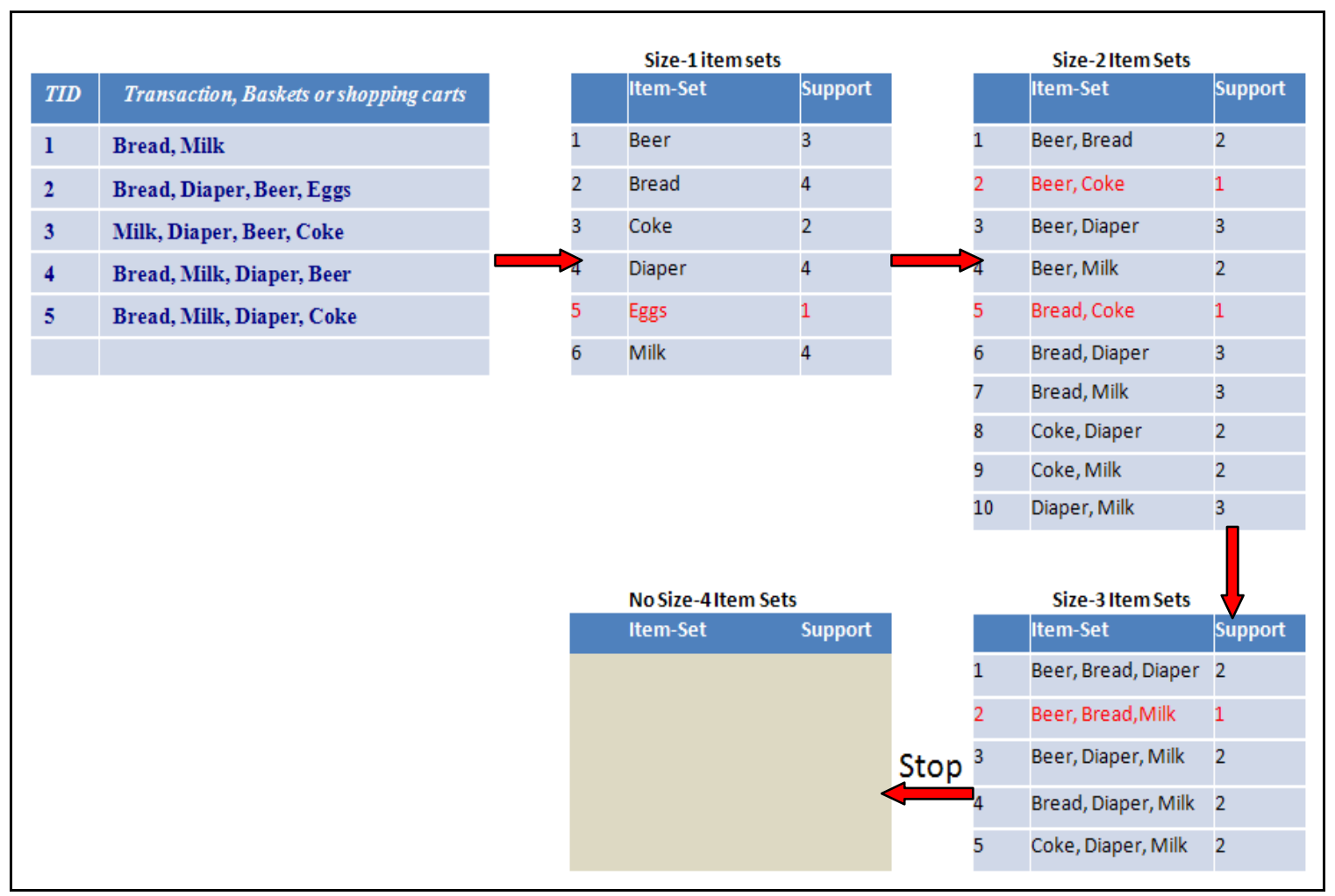

Figure 6: An Example Implementation of the Apriori Algorithm

\section{The Practice}

The Bikebuyers data set has 31,450 sales orders with 121,300 recorded items for 266 different products spanning 35 unique categories and 107 different models. Each record is a sales order that describes the details of the items sold (salesOrderNumber, quantity, productName, modelName, subcategoryName, categoryName). Figure 7 is a sample of the data set. According to the sample, SO43659 has 12 different products as follows: one "Mountain-100 Black, 42", three "Mountain-100 Black, 44"... and four "Sport-100 Helmet Blue".

After learning the theory, students use this large data set to perform market basket analysis. They use wizards to build and configure mining structures based on the "Model Name" of the items sold. The wizards allow students to configure the parameters, thresholds, and probabilities for item sets and association rules for the market basket analysis. Students can run multiple association analysis scenarios and analyze the item sets and their rules. Figure 8 is the output of a market basket analysis. It is composed of three tab-groups: (1) the association rules that were predicted, (2) the frequent item sets that were computed (detailed in Figure 11), and (3) the dependency network between the items. Figure 8 shows the output after executing a calibrated association analysis algorithm with minimum probability of 0.4 and importance threshold of 0.23 . The algorithm concluded that those who bought an "All-Purpose Bike Stand" and a "HL Road Tire" also bought a "Tire Tube" with a probability of 0.941 and an importance of 1.080. In other words, All- 
Purpose Bike Stand \& HL Road Tire $\rightarrow$ Road Tire Tube (0.941, 1.08).

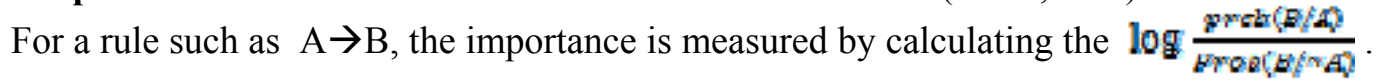

\begin{tabular}{|c|c|c|c|c|c|}
\hline 50 & Qty & ProductKane & HodelKane & SubCategoryliane & Categoryliane \\
\hline 5043659 & 1 & Mountain-100 Black, 42 & Mountain-100 & Mountain Bikes & Bikes \\
\hline 5043659 & & Mountain-100 Black, 44 & Mountain-100 & Mountain Bikes & Bikes \\
\hline 5043659 & & Mountain-100 Black, 48 & Mountain-100 & Mountain Bikes & Bikes \\
\hline 5043659 & & Mountain-100 Silver, 38 & Mountain-100 & Mountain Bikes & Bikes \\
\hline 5043659 & 1 & Mountain-100 Silver, 42 & Mountain-100 & Mountain Bikes & Bikes \\
\hline 5043659 & 2 & Mountain-100 Silver, 44 & Mountain-100 & Mountain Bikes & Bikes \\
\hline 5043659 & & Mountain-100 Silver, 48 & Mountain-100 & Mountain Bikes & Bikes \\
\hline 5043659 & 3 & Long-Sleeve Logo Jersey, M & Long-Sleeve Logo Jersey & Jerseys & Clothing \\
\hline 5043659 & & Long-Sleeve Logo Jersey, XI & Long-Sleeve Logo Jersey & Jerseys & Clothing \\
\hline 5043659 & 6 & Mountain Bike Socks, M & Mountain Bike Socks & Socks & Clothing \\
\hline 5043659 & 2 & AWC Logo Cap & Cycling Cap & Caps & Clothing \\
\hline 5043659 & 4 & Sport-100 Helmet, Blue & Sport-100 & Helmets & Accessories \\
\hline 5043660 & 1 & Road-650 Red, 44 & Road-650 & Road Bikes & Bikes \\
\hline 5043660 & & Road-450 Red, 52 & Road-450 & Road Bikes & Bikes \\
\hline 5043661 & & HL Mountain Frame - Black, 48 & HL Mountain Frame & Mountain Frames & Components \\
\hline 5043661 & & HL Mountain Frame - Black, 42 & HL Mountain Frame & Mountain Frames & Components \\
\hline 5043661 & 2 & HL Mountain Frame - Black, 38 & HL Mountain Frame & Mountain Frames & Components \\
\hline 5043661 & 4 & AWC Logo Cap & Cycling Cap & Caps & Clothing \\
\hline 5043661 & 4 & Long-Sleeve Logo Jersey, I & Long-Sleeve Logo Jersey & Jerseys & Clothing \\
\hline 5043661 & & HL Mountain Frame - Silver, 46 & HL Mountain Frame & Mountain Frames & Components \\
\hline 5043661 & 3 & Mountain-100 Black, 38 & Mountain-100 & Mountain Bikes & Bikes \\
\hline 5043661 & 2 & Mountain-100 Black, 48 & Mountain-100 & Mountain Bikes & Bikes \\
\hline 5043661 & 2 & Sport-100 Helmet, Blue & Sport-100 & Helmets & Accessories \\
\hline 5043661 & 2 & HL Mountain Frame - Silver, 48 & HL Mountain Frame & Mountain Frames & Components \\
\hline 5043661 & 4 & Mountain-100 Black, 42 & Mountain-100 & Mountain Bikes & Bikes \\
\hline 5043661 & 2 & Mountain-100 Silver, 44 & Mountain-100 & Mountain Bikes & Bikes \\
\hline 5043661 & 2 & Long-Sleeve Logo Jersey, XI & Long-Sleeve Logo Jersey & Jerseys & Clothing \\
\hline 5043661 & 2 & Mountain-100 Black, 44 & Mountain-100 & Mountain Bikes & Bikes \\
\hline 5043661 & 5 & Sport-100 Helmet, Black & Sport-100 & Helmets & Accessories \\
\hline 5043662 & 3 & Road-650 Red, 52 & Road-650 & Road Bikes & Bikes \\
\hline 5043662 & 5 & Road-650 Black, 52 & Road-650 & Road Bikes & Bikes \\
\hline 5043662 & 2 & LI Road Frame - Red, 62 & II Road Frame & Road Frames & Components \\
\hline 5043662 & 4 & Road-450 Red, 58 & Road-450 & Road Bikes & Bikes \\
\hline 5043662 & 3 & IL Road Frame - Red, 44 & LI Road Frame & Road Frames & Components \\
\hline 5043662 & 5 & Road-650 Red, 44 & Road-650 & Road Bikes & Bikes \\
\hline 5043662 & 3 & Road-650 Black, 58 & Road-650 & Road Bikes & Bikes \\
\hline 5043662 & 2 & Road-650 Black, 44 & Road-650 & Road Bikes & Bikes \\
\hline 5043662 & 1 & Road-150 Red, 56 & Road-150 & Road Bikes & Bikes \\
\hline 5043662 & & Road-450 Red, 44 & Road-450 & Road Bikes & Bikes \\
\hline 5043662 & 3 & Road-650 Red, 48 & Road-650 & Road Bikes & Bikes \\
\hline 5043662 & 1 & ML Road Frame - Red, 48 & ML Road Frame & Road Frames & Components \\
\hline 5043662 & 6 & Road-450 Red, 52 & Road-450 & Road Bikes & Bikes \\
\hline
\end{tabular}

Figure 7: A Sample of the Data Set

Figure 9 is an Excel export of the rules tab in Figure 8. Both the importance and probability of each rule are presented as a bar and a numeric value for validation, further analysis, sorting, filtering, etc. The user interface also allows an analyst to select a rule and drill through it to the record cases associated with that rule. Figure 10 is a drill through the top rule of Figure 8. Figure 11 is an Excel export of the item sets tab of Figure 8. Using the dependency network tab, students can also explore the item sets generated and the strength of dependencies between items. Students then store their results in work sheets for further data analysis. 


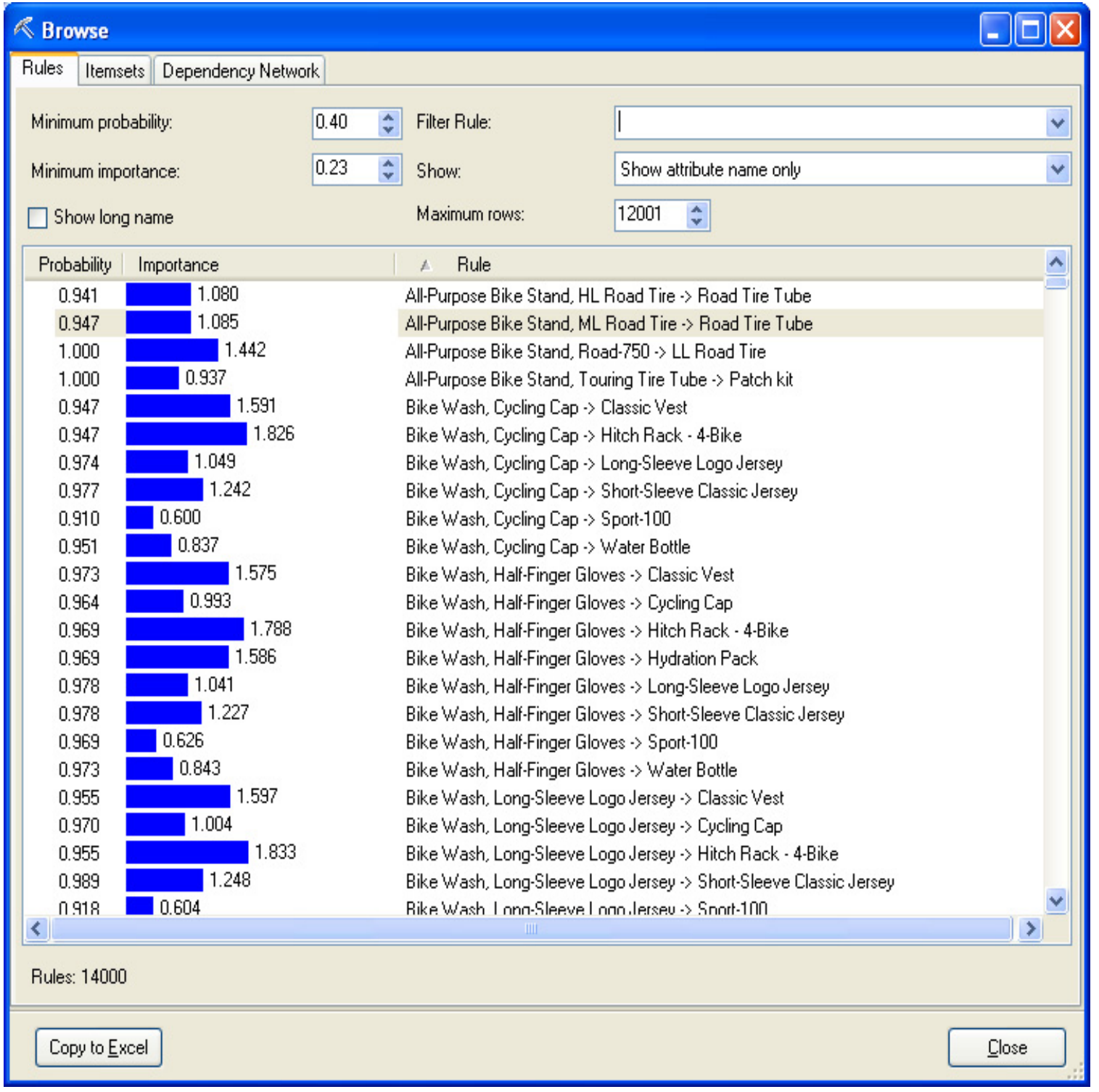

Figure 8: The Main Screen of the Market Basket Analysis 


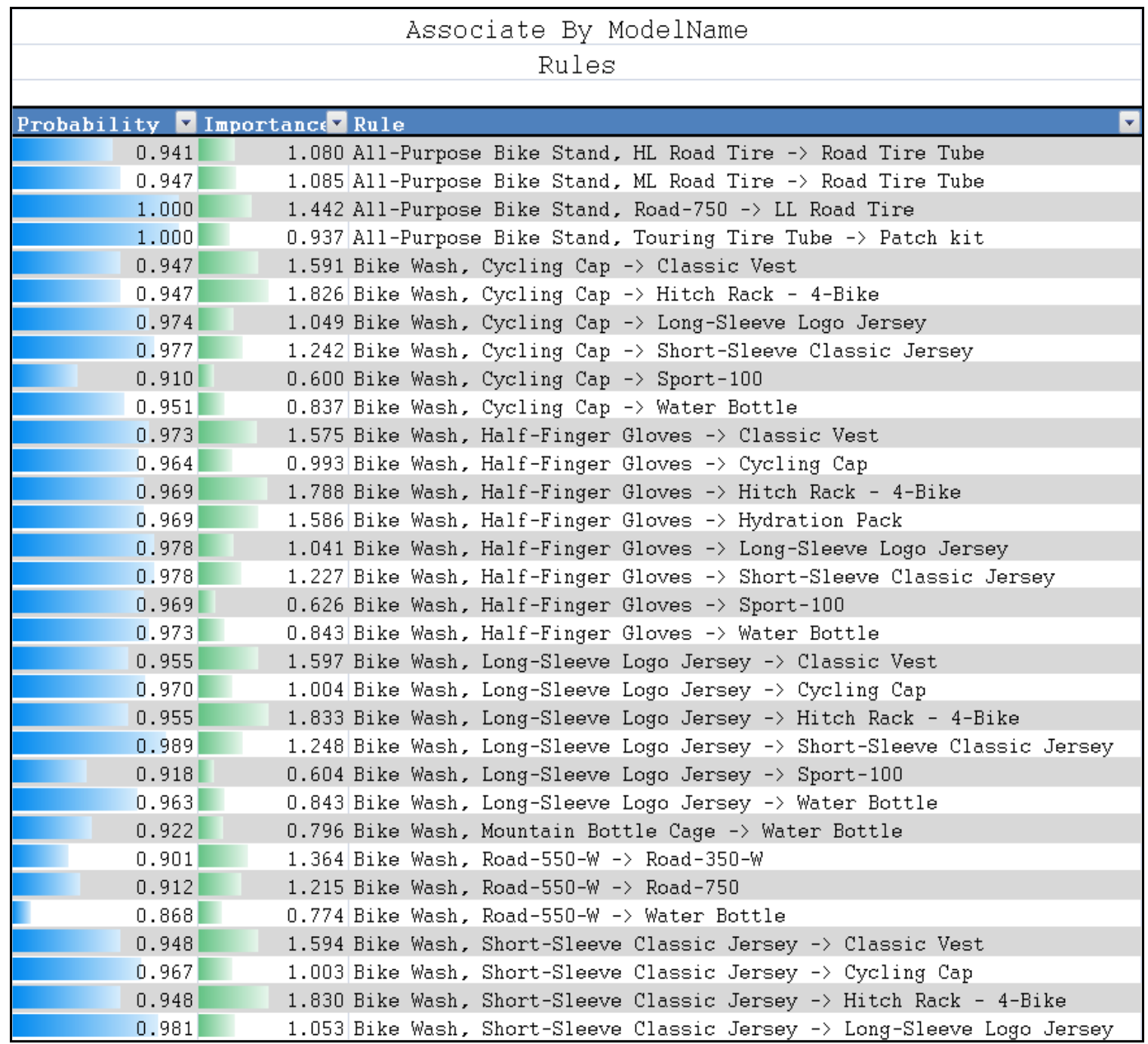

Figure 9: An Excel Export of the Rules Tab 


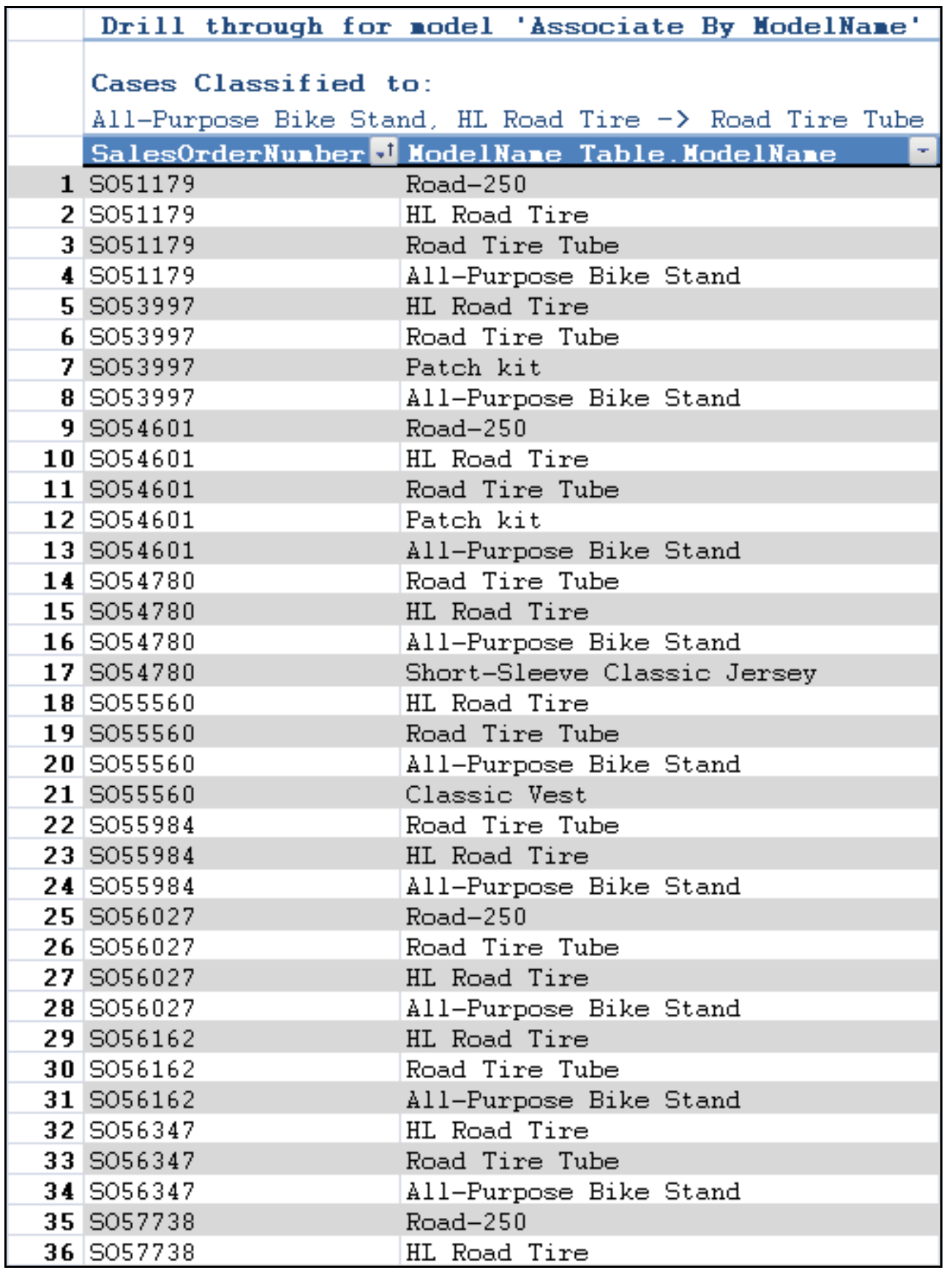

Figure 10: A Drill through the Top Association Rule Showing 36 out of 100 Cases 


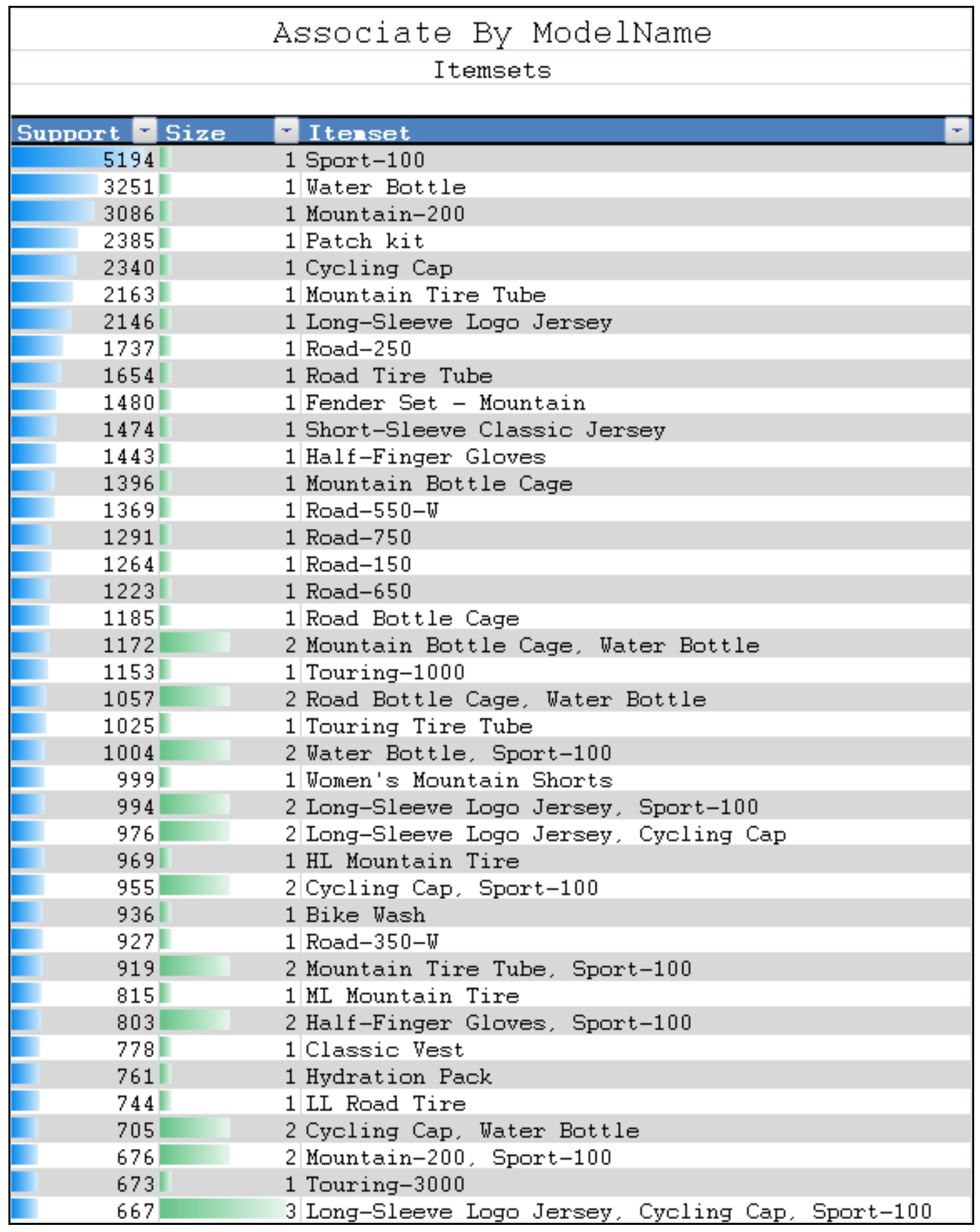

Figure 11: An Excel Export of the Item Sets Tab 


\section{Classification and Prediction}

Classification and prediction is the most elaborate part of a data mining course. "Classification is the task of learning a target function that maps each attribute set $X$ to one of the predefined class labels Y" (Tan et al., 2006). In an introductory course in data mining, students learn decision trees, naïve Bayes, neural networks, and logistic regression models.

Classification and prediction is a five-step process: (1) select a classification algorithm, (2) calibrate the parameters of the algorithm, (3) feed a training data set to the algorithm to learn a classification model, (4) load a test data set to the learned model to measure its accuracy, (5) use the learned model to predict previously unknown classifications. Model fitting is an iterative process (steps 2, 3, and 4). Algorithm parameters are calibrated and fine-tuned during the process of finding a satisfactory mining model. Through confusion matrices and accuracy charts, students compare the performance of various mining models to select an appropriate model.

\section{The Theory}

Students learn the theoretical foundation and concepts of classification analysis through book chapters, lecture notes, lectures, and homework assignments. In our course, we cover the following areas of classification and prediction:

- Decision tree algorithms: Students learn information gain concepts, best entry selection for a tree-split, entropy, Gini index, and classification error measures.

- Naïve Bayes algorithms: Students learn conditional, prior and posterior probability, independence, and correlation between attributes.

- Neural networks algorithms: Students learn the "simple" concepts of back propagation, nodes, and layers (the mathematical theory of neural networks is beyond the scope of the course).

- Logistic regression analysis: Students learn the difference between a standard linear regression and a logistic regression.

\section{The Practice}

For the practice, we used the Mushrooms data set from the UCI repository (University of California Irvine, 2009). Figure 12 is a partial sample of the data set. The class column is the classification column with values edible or poisonous.

First students perform elementary data analysis on the data set. They explore (1) the characteristics of the attributes and their sets of legal values, and (2) elementary classifications, histograms, and groupings using pivot tables and pivot charts. Figure 13 is a pivot chart of the profiles of some of the attributes. It shows the distribution of the classification of a mushroom broken down by attribute type and value. The second row indicates that the data set has 3343 records of:

bruises $=$ none mushrooms with

prob(bruises $=$ none/edible $)=0.35 \&$

prob(bruises $=$ bruises $/$ poisonous $)=0.84$ 


\begin{tabular}{|c|c|c|c|c|c|c|c|c|c|c|}
\hline ID & $\checkmark$ class $\vee$ & capShape & capSurface & capColor & bruises & odor & $\checkmark$ gillAttachment & gillSpacing & gillSize & gillColor $\mathrm{v}$ \\
\hline & 1 poisonous & usflat & scaly & brown & none & fishy & free & close & narrow & buff \\
\hline & 2 poisonous & us convex & smooth & buff & bruises & foul & free & close & broad & chocolate \\
\hline & 3 edible & convex & scaly & brown & bruises & almond & free & close & broad & white \\
\hline & 4 edible & convex & scaly & brown & bruises & none & free & close & broad & white \\
\hline & 5 edible & knobbed & smooth & brown & none & none & attached & close & broad & yellow \\
\hline & 6 poisonous & us knobbed & scaly & red & none & spicey & free & close & narrow & buff \\
\hline & 7 edible & bell & smooth & white & none & none & free & crowded & broad & gray \\
\hline & 8 poisonous & us flat & smooth & white & bruises & pungent & $t$ free & close & narrow & black \\
\hline & 9 poisonous & usflat & fibrous & yellow & none & foul & free & close & broad & chocolate \\
\hline & 10 edible & flat & fibrous & brown & bruises & none & free & close & broad & brown \\
\hline & 11 edible & flat & fibrous & gray & none & none & free & crowded & broad & brown \\
\hline & 12 edible & flat & fibrous & white & none & none & free & crowded & broad & brown \\
\hline & 13 edible & flat & smooth & brown & none & none & free & close & broad & white \\
\hline & 14 edible & flat & fibrous & brown & bruises & none & free & close & broad & white \\
\hline & 15 edible & bell & smooth & brown & none & none & attached & close & broad & orange \\
\hline & 16 poisonous & us knobbed & scaly & red & none & fishy & free & close & narrow & buff \\
\hline & 17 edible & flat & fibrous & brown & none & none & free & crowded & broad & chocolate \\
\hline
\end{tabular}

Figure 12: Sample Records of the Mushroom Data Set

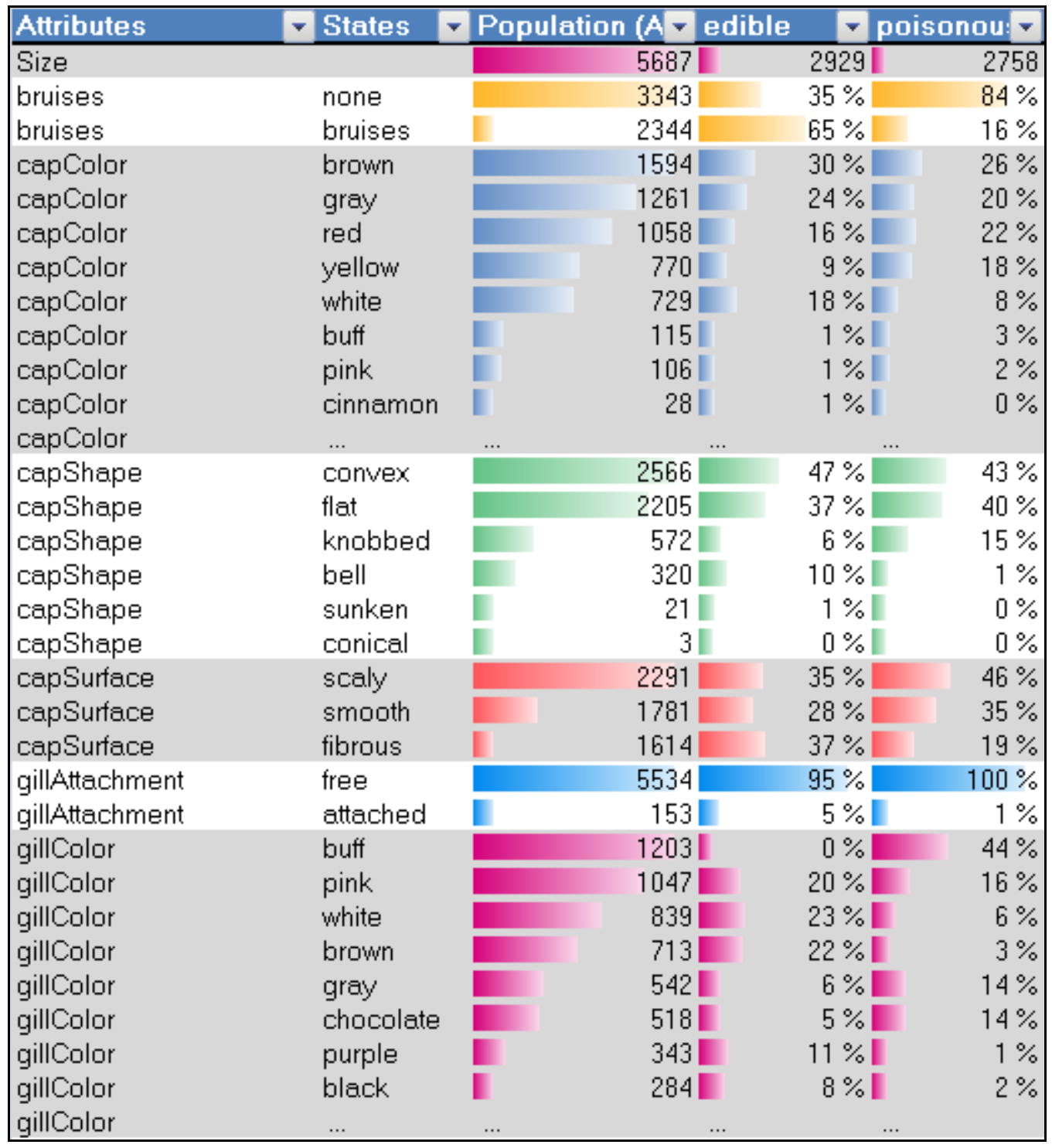

Figure 13: Sample Attribute Profiles of the Data Set 
For the purpose of classification analysis, we built a mining structure and four mining models (a decision tree model, a naïve Bayes model, a neural network model, and a logistic regression model). Then we compared the performance of these models using the holdout set, accuracy charts, and a classification matrix.

\section{The mining structure}

Students used wizards to create and configure a mining structure. This involved (1) the inclusion and exclusion of attributes, (2) the configuration of the characteristics of each attribute (key, data type, content type) and (3) the split percentage of data into training and testing (holdout) sets.

\section{Decision tree mining model.}

To build a decision tree model, students configured parameters support such as information gain, scoring methods, and type of tree split method. Data and configuration are then sent to the data mining computing engine. A decision tree-mining model with drill through, legends, and display capabilities for each node is generated and sent back to Excel. Figure 14 is an example of a decision tree that has been generated. Students can view the details of the mining legend of each node and drill through it to the underlying data set that supported that node. For example, tracing the "stalkSurfaceBelowRing not = 'scaly"' node, the classification rule is:

If $\quad$ odor $=$ 'none' $\boldsymbol{\&}$ sporePrintColor $=$ 'white' $\boldsymbol{\&}$

ringNumber $!=2 \&$ stalkSurfaceBelowRing != 'scaly'

Then Prob(mushroom is edible) $=85.6 \&$

Prob(mushroom is poisonous $)=14.4$

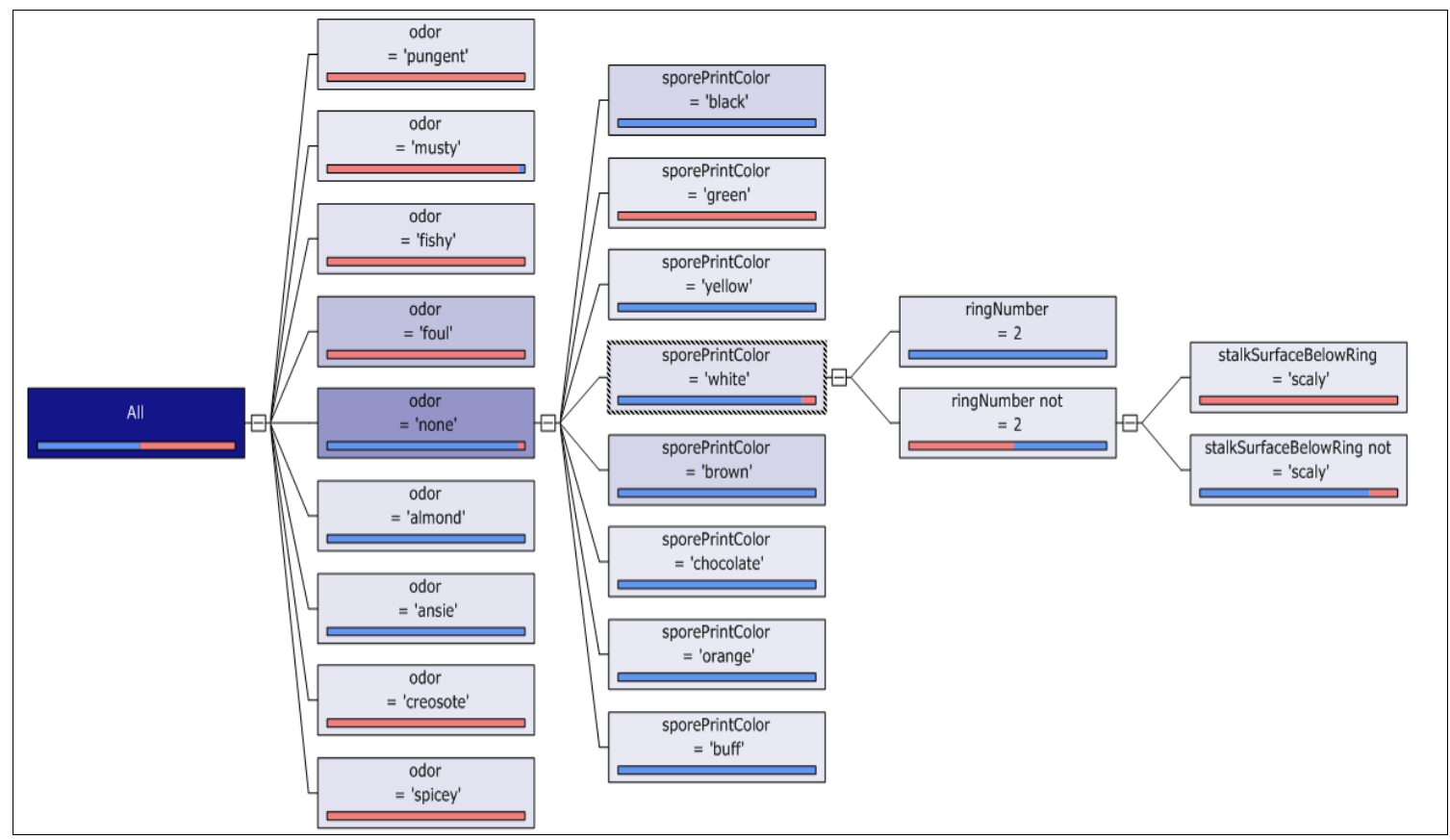

Figure 14: A Decision Tree Classification of the Mushroom Data Set

\section{Naïve Bayes, logistic regression and neural network models}

Similarly, students used wizards to configure parameters and to build a naïve Bayes, a logistic regression, and a neural network classification model. The models display discrimination tables that show each attribute value, the classification it favors, and a bar chart as a measure of support. Figure 15 is the naïve Bayes output for the same mining structure as in the decision tree. It dis- 
plays the attributes, their values, and the level of contribution of each value to the favored classification. Figure 16 is the logistic regression model output, and Figure 17 is the neural network model output of the same mining structure. It is also possible to build multiple decision tree mining models with different calibrations and attributes and then compare the models for accuracy and validity.

\begin{tabular}{|c|c|c|c|}
\hline Attributes & Values & Favors edible & Favors poisonous \\
\hline odor & none & & \\
\hline odor & foul & & \\
\hline stalkSurfaceAboveRing & g silky & & \\
\hline gillColor & buff & & \\
\hline gillSize & broad & & \\
\hline gillSize & narrow & & \\
\hline ring Type & pendant & & \\
\hline sporePrintColor & chocolate & & 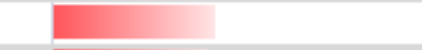 \\
\hline ringType & large & & \\
\hline bruises & none & & \\
\hline bruises & bruises & & \\
\hline stalkSurfaceAboveRing & s smooth & - & \\
\hline population & several & & \\
\hline sporePrintColor & brown & 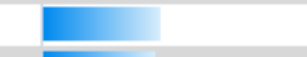 & \\
\hline sporePrintColor & black & 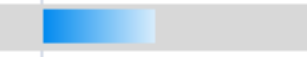 & \\
\hline gillSpacing & close & & 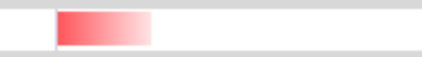 \\
\hline gillSpacing & crowded & L & \\
\hline sporePrintColor & white & & 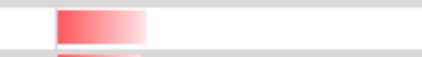 \\
\hline habitat & paths & & 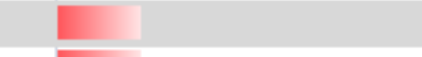 \\
\hline odor & spicey & & 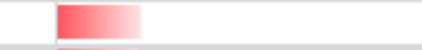 \\
\hline odor & fishy & & \\
\hline
\end{tabular}

Figure 15: Naïve Bayes: Attribute Discrimination of Class

\begin{tabular}{|l|l|l|l|}
\hline Attribute & Value & Favors poisonous & F Favors edible \\
\hline sporePrintColor & green & & \\
ringType & flaring & & \\
\hline odor & creosote & & \\
\hline odor & pungent & & \\
stalkColorAboveRing & yellow & & \\
stalkColorBelowRing & yellow & & \\
sporePrintColor & purple & & \\
capShape & sunken & & \\
capShape & conical & & \\
\hline odor & musty & & \\
capColor & cinnamon & & \\
sporePrintColor & buff & & \\
\hline odor & foul & & \\
sporePrintColor & chocolate & & \\
capColor & purple & & \\
stalkRoot & rooted & & \\
odor & none & & \\
\hline
\end{tabular}

Figure 16: Logistic Regression: Attribute Discrimination of class 


\begin{tabular}{|c|c|c|c|}
\hline Attribute & Value & Favors edible & Favors poisonous \\
\hline sporePrintColor & green & & \\
\hline odor & creosote & & \\
\hline odor & pungent & & \\
\hline gillColor & green & & \\
\hline ring Type & flaring & & \\
\hline capColor & green & & \\
\hline odor & foul & & \\
\hline sporePrintColor & purple & & \\
\hline stalkColorAboveRing & yellow & & \\
\hline stalkColorBelowRing & orange & & \\
\hline capShape & sunken & & \\
\hline odor & none & & \\
\hline stalkColorBelowRing & yellow & & \\
\hline capColor & cinnamon & & \\
\hline ringNumber & 2 & & \\
\hline population & abundant & & \\
\hline population & numerous & & \\
\hline capColor & purple & & \\
\hline ringType & none & & \\
\hline
\end{tabular}

Figure 17: Neural Network: Attribute discrimination of Class

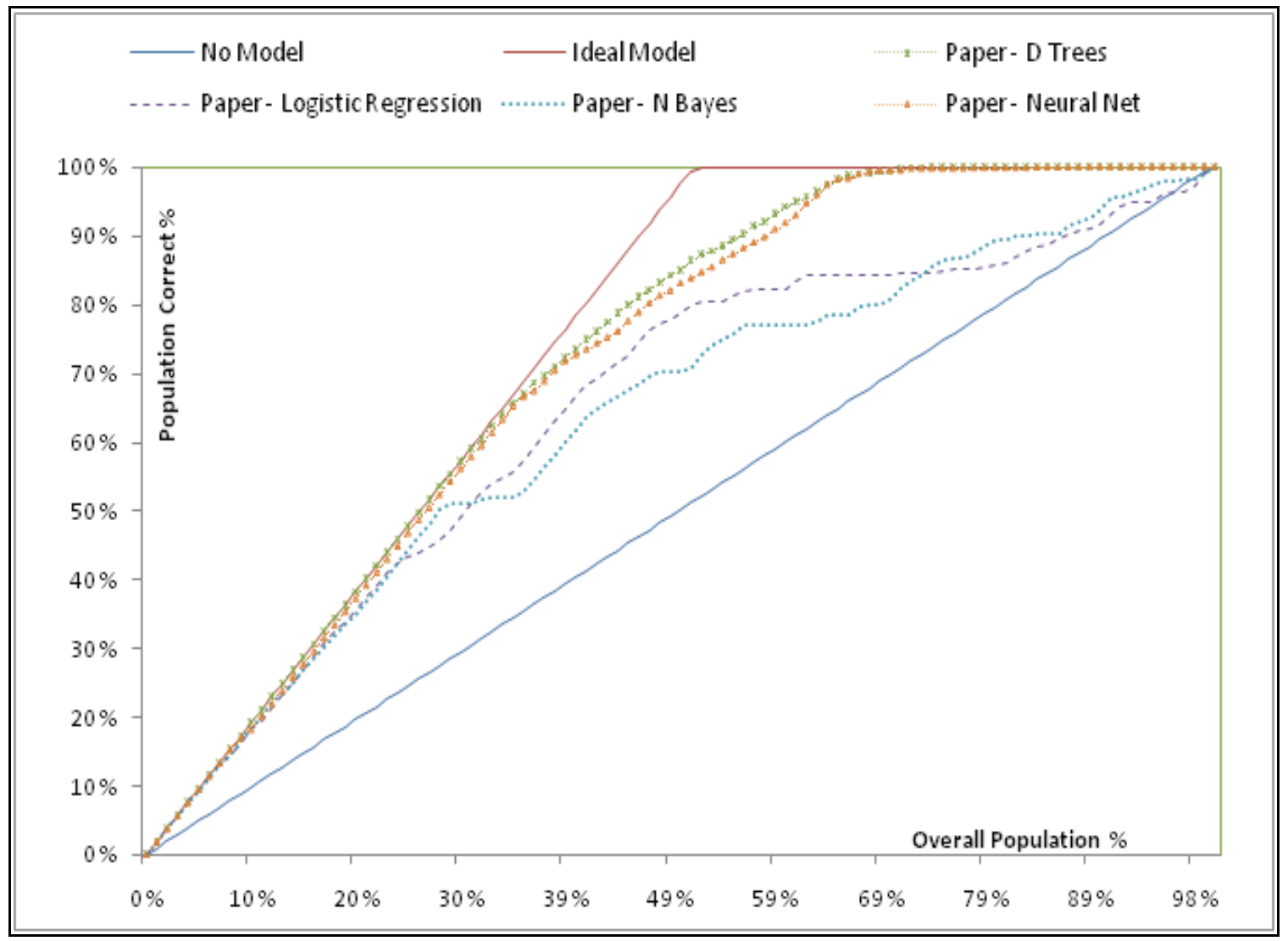

Figure 18: Accuracy (Lift) Chart of 4 the Classification Models for the Poisonous class 


\section{Model validation}

We demonstrated the simplicity of building a classification mining structure and four mining models for that mining structure. We would like to measure the accuracy of each mining model and compare their performance. Students use wizards to build accuracy charts for each of the four mining models. Figure 18 is the output of such a process. The straight-line from the origin $(0,0)$ to $(100 \%, 100 \%)$ is the random predictor model. The broken-line from $(0,0)$ to $(49 \%, 100 \%)$ to $(100 \%, 100 \%)$ is the ideal model predictor. The ideal predictor will correctly predict every classification. From the graph, the ideal line implies that $49 \%$ of the mushrooms in the testing data set are poisonous. The other curves are the decision tree (close to the ideal line), the neural network, the logistic regression, and the naïve Bayes model predictors. Analyzing the chart, the decision tree outperformed the rest of the models. The naïve Bayes model performed worse than the rest of the three models.

\section{Cluster Analysis}

Finally, students learn how to perform cluster analysis of data, which is a form of unsupervised learning and grouping of data records.

\section{The Theory}

Students learn the theoretical foundation and concepts of cluster analysis through book chapters, lecture notes, lectures, and homework assignments. The topics covered include: (1) distance and weighted distance between objects, (2) similarity measures and weighted similarity measures between data types (nominal, ordinal, interval, and ratio), (3) various k-norms ( $\mathrm{k}=1,2$ and $\infty$ ), (4) simple matching coefficient, cosine, Jacard, and correlation, (5) various center-based clustering algorithms such as the k-means, bisection k-means, and (6) density-based clustering algorithms such as DBSCAN.

\section{The Practice}

For the practice, we used the Iris and the Mushrooms data sets. The Iris data set provides an allnumeric ratio scale measure. The Mushrooms data set provides an all-qualitative categorical scale measure. Using wizards, students configured the attributes of interest the maximum number of clusters, the split methods, clustering algorithm to use, and minimum cluster size. Figure 19 is the output of a clustering run. The characteristics of each predicted category is displayed. The relative importance of the range of values of an attribute to the classification of the category is presented as a bar chart. The longer the bar, the stronger the relative importance of the corresponding attribute value for that category.

Students also learn how to perform classification through hierarchical clustering of data. From Figure 20 students could see that category 2 and 3 are well clustered around the setosa and the versicolor irises. However, category 1 has a mix of versicolor (14 records) and virginica (50 records) irises. Students then use category 1 records to perform more clustering on the records of this category to extract clear separation criterion between the clusters.

Similarly, we performed (auto detect) hierarchical clustering analysis against the Mushrooms data set. Nine categories or clusters were detected. Mapping the clusters against the poisonous and edible classifications, categories 1, 2, 3, and 5 produced a perfect fit. Figure 21 shows the classification matrix of the first iteration of the cluster analysis. 


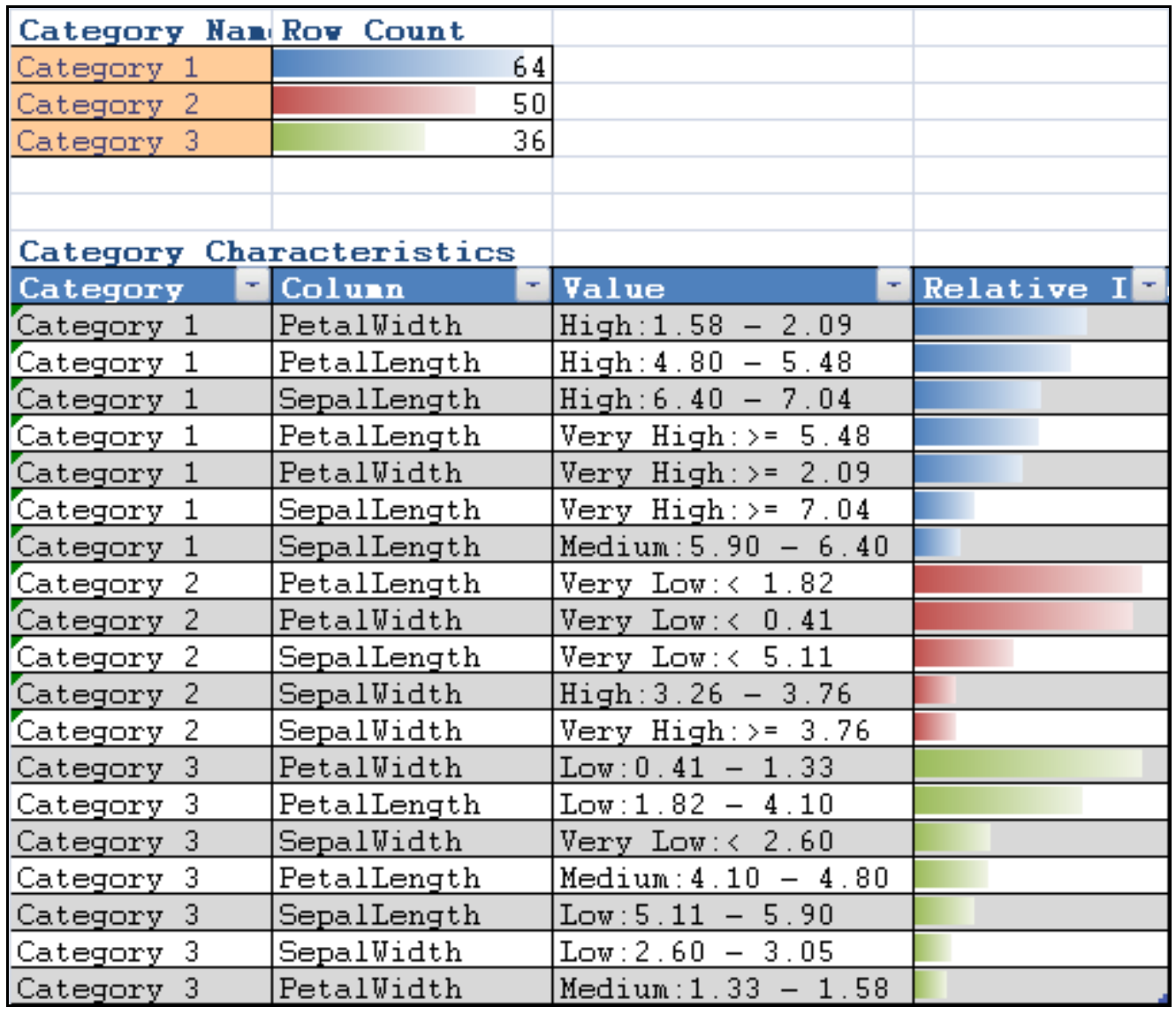

Figure 19: Category Characteristics of Iris Data

\begin{tabular}{|l|r|r|r|r|}
\hline Count of id & - & & \\
\hline Rov Labels & Iris-setosa & Iris-versicolor & Iris-virginica & Total \\
\hline Category 1 & & 14 & 50 & 64 \\
\hline Category 2 & 50 & 36 & & 50 \\
\hline Category 3 & & $\mathbf{5 0}$ & $\mathbf{5 0}$ & $\mathbf{1 5 0}$ \\
\hline Total & $\mathbf{5 0}$ & $\mathbf{5 0}$ & \\
\hline
\end{tabular}

Figure 20: Accuracy Clustering Matrix

\begin{tabular}{|c|c|c|c|}
\hline Categories - & edible & poisonous & Grand Total \\
\hline Category 1 & & 1728 & 1728 \\
\hline Category 2 & 1728 & & 1728 \\
\hline Category 3 & & 1296 & 1296 \\
\hline Category 4 & 704 & 256 & 960 \\
\hline Category 5 & 864 & & 864 \\
\hline Category 6 & 96 & 480 & 576 \\
\hline Category 7 & 320 & 72 & 392 \\
\hline Category 8 & 304 & 48 & 352 \\
\hline Category 9 & 192 & 36 & 228 \\
\hline Grand Total & 4208 & 3916 & 8124 \\
\hline
\end{tabular}

Figure 21: Mapping Detected Categories to Classifications 
Figure 22 shows the characteristics of each cluster. Categories 1 and 3 produced poisonous classifications while categories 2 and 5 produced edible classifications. Excel add-ins allow us to relabel category names. For example, we could re-label category 1 as poisonous- 1 . The records of categories 1,2,3, and 5 were filtered out and another classification was performed on the records of the remaining categories. Two iterations later, a perfect match was produced.

\begin{tabular}{|c|c|c|c|}
\hline Category & Column & Value $\quad-1$ & |Relative Importance \\
\hline Category 1 & gillColor & buff & \\
\hline Category 1 & sporePrintColor & white & \\
\hline Category 1 & stalkRoot & cup & \\
\hline Category 1 & gillSize & narrow & \\
\hline Category 1 & ring Type & evanescent & \\
\hline Category 1 & population & several & \\
\hline Category 1 & stalkShape & tapering & \\
\hline Category 1 & bruises & none & \\
\hline Category 1 & odor & spicey & \\
\hline Category 1 & odor & fishy & \\
\hline Category 2 & habitat & woods & \\
\hline Category 2 & bruises & bruises & \\
\hline Category 2 & odor & none & \\
\hline Category 2 & stalkRoot & bulbuous & \\
\hline Category 2 & ringType & pendant & \\
\hline Category 2 & stalkShape & tapering & \\
\hline Category 2 & stalkColorAboveRing & gray & \\
\hline Category 2 & stalkColorBelowRing & gray & \\
\hline Category 2 & stalkSurfaceAboveRing & smooth & \\
\hline Category 2 & gillSize & broad & \\
\hline Category 2 & gillColor & purple & \\
\hline Category 2 & population & solitary & $=$ \\
\hline Category 2 & sporePrintColor & black & \\
\hline Category 3 & ringType & large & \\
\hline Category 3 & sporePrintColor & chocolate & \\
\hline Category 3 & odor & foul & \\
\hline Category 3 & stalkSurfaceAboveRing & $\begin{array}{ll}9 & \text { silky } \\
\end{array}$ & \\
\hline Category 3 & stalkShape & enlarging & \\
\hline Category 3 & stalkRoot & bulbuous & \\
\hline Category 3 & stalkColorAboveRing & buff & \\
\hline Category 3 & stalkColorBelowRing & buff & \\
\hline Category 3 & stalkColorAboveRing & brown & . \\
\hline Category 3 & bruises & none & \\
\hline Category 5 & stalkRoot & equal & \\
\hline Category 5 & gillSpacing & crowded & \\
\hline Category 5 & population & abundant & \\
\hline Category 5 & habitat & grasses & \\
\hline Category 5 & odor & none & \\
\hline Category 5 & ringType & evanescent & \\
\hline Category 5 & stalkSurfaceAboveRing & a fibrous & \\
\hline Category 5 & stalkSurfaceBelowRing & fibrous & \\
\hline Category 5 & stalkColorBelowRing & white & \\
\hline Category 5 & stalkColorAboveRing & white & \\
\hline Category 5 & bruises & none & \\
\hline
\end{tabular}

Figure 22: Characteristics of the three Clusters that Produced a Perfect Match 


\section{Summary and Conclusions}

Data mining and data analysis for the purpose of decision support is a fast growing area of computing. In the early 2000s, a data mining methods course was taught as a pure research topic in Computer Science. With the maturity of the discipline, the convergence of the data mining algorithms and the availability of computing platforms, students can now learn data mining methods as a problem solving discipline. The theory has matured. Standard textbooks are published. Accompanying technologies that implement the same basic algorithms are available at nominal costs through academic initiatives from companies like Oracle, IBM, and Microsoft. Accordingly, Information Systems programs are capable of providing a computing platform in support of their data mining methods courses. We strongly recommend extending the Information Systems curriculum to include a data mining track of two or more courses.

Walstrom, Schmbach, \& Crampton (2008) provided an in-depth survey of 300 students enrolled in an introductory business course justifying their reasons for not choosing Information Systems as an area of specialization. We do see a track in data mining methods enhances the career opportunities of Information Systems students. It is a sustainable growth area that is natural to an Information Systems program. Information Systems students should be able to represent, consolidate, and analyze data using data mining tools to provide organizations with business intelligence for the purpose of decision support.

The content presented and the hands on experience gained have broader applications. In the past, the authors have taught the Introduction to Management Information Systems course. Accounting, finance, general business, and marketing students benefited from the market basket analysis and the decision tree homework assignments. It was easy for the students to analyze large sets of data and produce business intelligence reports.

Finally, if it was not for the underlying technologies that we used, it would have been impossible to cover such material in a one-semester course and provide students with much needed hands-on experience in data mining. What we presented is how to utilize the cloud as a computing platform that transformed the role of a student from "doing low-level IT" in a data mining course to a business intelligence analyst using tools to analyze data for the purpose of decision support (Markoff, 2009).

\section{References}

Campos, M. M., Stengard, P. J., \& Milenova, B. L. (2005). Data-centric automated data mining. Proceedings of the Fourth International Conference on Machine Learning and Applications. IEEE Computer Society, 97-104.

Goharian, N., Grossman, D., \& Raju, N. (2004). Extending the undergraduate computer science curriculum to include data mining. Proceeding of the International Conference on Information Technology: Coding and Computing, 2, P.251.

Han, J., \& Kamber, M. (2006). Data mining concepts and techniques. Boston: Elsevier.

Jafar, M. J., Anderson, R. R., \& Abdullat, A. (2008a). Data mining methods course for computer information systems students. Information Systems Education Journal, 6(48). Retrieved from http://isedj.org/6/48/ISEDJ.6\%2848\%29.Jafar.pdf

Jafar, M. J., Anderson, R. R., \& Abdullat, A. (2008b). Software academic initiatives: A framework for supporting a contemporary information systems academic curriculum. Information Systems Education Journal, 6(55). Retrieved from http://isedj.org/6/55/index.html

King, M. A. (2009). A realistic data warehouse project: An integration of Microsoft Access and Microsoft Excel advanced features and skills. Journal of Information Technology Education, 8, IIP-091-104. Retrieved from http://www.jite.org/documents/Vol8/JITEv8IIP091-104King696.pdf 
Lenox, T. L., \& Cuff, C. (2002). Development of a data mining course for undergraduate students. Proceedings of the Information Systems Education Conference, 19.

Musicant, D. R. (2006). A data mining course for computer science: Primary sources and implementations. 37th SIGCSE technical symposium on Computer Science Education. ACM, 538-542.

Markoff, J. (2009, Dec. 14). A deluge of data shapes a new era of computing. The New York Times. Retrieved Dec. 14, 2009, from http://www.nytimes.com/2009/12/15/science/15books.html?_r=1\&scp=3\&sq=cloud\%20computing\%2 $\underline{0 \text { microsoft\&st }=\text { cse }}$

Rahal, I. (2008). Undergraduate research experiences in data mining. 39th SIGCSE Technical Symposium on Computer Science Education, ACM, 461-465.

Saquer, J. (2007). A data mining course for computer science and non-computer science students. Journal of Computing Sciences in Colleges, 22(4) 109-114.

Tan, P., Steinbach, M., \& Kumar, V. (2006). Introduction to data mining. Boston: Pearson Education.

Tang, H. (2008). A simple approach of data mining in Excel. 4th International Conference on Wireless Communication, Networking and Mobile Computing, 1-4.

University of California Irvine. (2009). UCI machine learning repository. Retrieved May 10, 2009, from http://archive.ics.uci.edu/ml/

Walstrom, K. A., Schmbach, T. P., \& Crampton, W. J. (2008). Why are students not majoring in information systems? Journal of Information Systems Education, 19(1), 43-52.

\section{Biography}

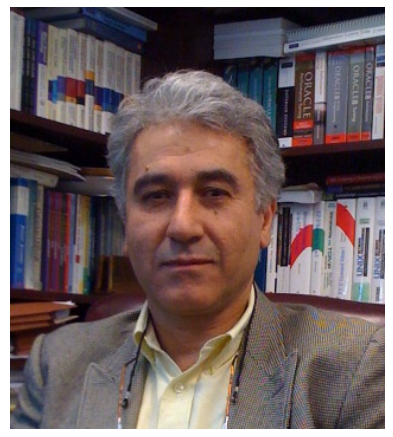

Musa J. Jafar is Gensler Professor of Computer Information Systems at West Texas A\&M University. He holds a Ph.D. in Systems Engineering, an M.S. in Management Information Systems, and an M.S. in Systems Engineering from the University of Arizona at Tucson. He also holds an M.S. in Mathematics from the American University of Beirut. He worked as a senior software engineer and architect for IBM, U.S. West Communications and Bell Communications Research. Additional information can be found at: http://faculty.cis.wtamu.edu/jafar/ 